\title{
TLR4/MyD88 signaling determines the metastatic potential of breast cancer cells
}

\author{
KUNLIN WU ${ }^{1}$, HUIHAO ZHANG ${ }^{1}$, YAJUAN FU ${ }^{2}$, YOUZHI ZHU ${ }^{1}$, LINGJUN KONG ${ }^{1}$, \\ LING CHEN $^{1}$, FENG ZHAO ${ }^{3}$, LIANGFEI YU ${ }^{3}$ and XIANGJIN CHEN ${ }^{1}$ \\ ${ }^{1}$ Department of Thyroid and Breast Surgery, The First Affiliated Hospital of Fujian Medical University, Fuzhou, \\ Fujian 350005; ${ }^{2}$ Southern Biomedical Research Center, Fujian Normal University, Fuzhou, Fujian 350007; \\ ${ }^{3}$ First Clinical Medical College, Fujian Medical University, Fuzhou, Fujian 350004, P.R. China
}

Received August 23, 2017; Accepted June 12, 2018

DOI: $10.3892 / \mathrm{mmr} .2018 .9326$

\begin{abstract}
The influence of Toll-like receptor (TLR)4/myeloid differentiation factor (MyD)88 signaling on the invasion and metastasis of cancer cells has been previously reported. The purpose of the present study was to determine the role of TLR4/MyD88 in breast cancer cell migration and invasion, and to discover novel therapeutic targets for breast cancer treatment. TLR4, MyD88 and high mobility group box 1 (HMGB1) mRNA expression levels were assessed in highly invasive human MDA-MB-231 breast cancer cells, breast cancer cells with a low rate of invasion (MCF-7) and normal human MDA-Kb2 mammary gland cells by reverse transcription-quantitative polymerase chain reaction. The protein expression levels of these markers were detected by western blotting and immunofluorescence. Randomly selected breast cancer and paracarcinoma tissues were used to measure TLR4 and MyD88 protein expression levels by immunohistochemistry. The mRNA and protein expression levels of TLR4 and MyD88 were significantly higher in MDA-MB-231 cells compared with either MCF-7 cells or MDA-Kb2 cells. The mRNA and protein expression levels of HMGB1 were comparable in the two breast cancer cell lines, with no statistical difference ( $>>0.05)$. TLR4 and MyD88 protein expression levels were also significantly higher in breast cancer tissues compared with paracarcinoma tissues $(\mathrm{P}<0.05)$. TLR4 and MyD88 protein expression levels were positively correlated with axillary lymph node metastasis and histological grade $(\mathrm{P}<0.05)$. TLR4/MyD88 expression levels were positively correlated with the metastasis of breast cancer cells. TLR4/MyD88 may be useful as a novel biomarker to
\end{abstract}

Correspondence to: Dr Xiangjin Chen, Department of Thyroid and Breast Surgery, The First Affiliated Hospital of Fujian Medical University, 20 Chazhong Road, Chating Street, Fuzhou, Fujian 350005, P.R. China

E-mail: chenxiangjin_c@sina.com

Key words: breast cancer, invasiveness, Toll-like receptor 4, myeloid differentiation factor 88 , high mobility group box 1 evaluate the prognosis and treatment of patients with breast cancer.

\section{Introduction}

Of all gynecological diseases, breast cancer has the highest incidence worldwide (1). There are a number of causes of breast cancer, including aberrant estrogen receptor signaling (2), genetic and environmental factors (3-5) and inflammation (6). However, breast cancer is not as invasive as other gynecological tumors, including cervical and ovarian cancers. There are two types of breast cancer cell lines (MDA-MB-231 and MCF-7) that are frequently used as models of breast cancer progression and which exhibit varying metastatic potential (7). Multiple different signaling pathways control the metastatic potential of these cells, including those involved in growth and apoptosis $(8,9)$, hypoxia-associated gene expression (10), proteasomal activation and serine/threonine-protein kinase mTOR (mTOR) (11), protein Wnt5a (12), phosphatidylinositol 3-kinase (13), nuclear factor- $\kappa \mathrm{B}(\mathrm{NF}-\kappa \mathrm{B})(14)$ and nuclear factor erythroid 2-related factor 2 signaling (15). These pathways may also contribute to resistance against first-line endocrine therapy (16).

Toll-like receptors (TLRs) are ancient microbial pattern recognition receptors that are highly conserved from Drosophila to humans. TLR4 activates myeloid differentiation factor 88 (MyD88) upon receiving tumor antigen information and promotes the resting state of NF- $\mathrm{B}$ nuclear translocation, finally activating gene transcription (17). By contrast, TLR4 may also allow tumor cells to escape host immune surveillance through the MyD88 signaling pathway. Li et al (18) identified that high expression levels of TLR4 and MyD88 were associated with poor overall survival rates in patients with epithelial ovarian cancer (EOC). Inhibition of TLR4/MyD88 signaling may therefore be a useful tool in promoting DNA repair and maintaining immune responses following ultraviolet radiation-induced damage, which contributes to the development of nonmelanoma skin cancer (19). High levels of MyD88 are also associated with reduced survival rates of patients with EOC (20). Atractylenolide-I, a novel TLR4-antagonist, inhibits lymphocyte antigen 96 (MD-2)-mediated TLR4/MyD88 signaling, making it a potential therapy for patients with EOC (21). Finally, targeting the cyclooxygenase 2/prostaglandin 
E2 and TLR/MyD88 signaling pathways in gastric cancer cells suppresses inflammation and maintains stemness (22).

High mobility group box 1 (HMGBl), an endogenous ligand for TLR4, has attracted much attention in recent years. HMGB1 is an abundant non-histone nuclear transcription factor and is involved in the growth and metastasis of prostate (23), colorectal (24), gastric (25), liver (26) and lung (27) tumors. TLR4 acts as a transmembrane receptor that is able to activate MyD88-dependent signaling in response to the binding of HMGB1. HMGB1-mediated TLR4/MyD88 signaling has been implicated in the invasion and metastasis of a number of different cancer cell types $(18,19)$. However, the role of TLR4/MyD88 in human breast cancer progression has not been well characterized.

A previous study identified that the mRNA expression levels of TLR4 and MYD88 were significantly higher in breast cancer cells compared with fibroadenoma cells and adjacent normal tissues; high protein expression levels of TLR4 and MyD88 were also associated with poor clinical prognosis (28). The current study aimed to examine the mechanisms underlying cancer cell invasion mediated by TLR4 and MyD88. MDA-MB-231 and MCF-7 represent human breast cell lines with varying metastatic and invasive potential. Generally, MCF-7 cells are non-invasive, while MDA-MB-231 cells are highly invasive (29) and used to examine the mechanisms of breast cancer metastasis (30). The present study used these two cellular models of invasion to examine the association between TLR4, MyD88 and HMGB1 expression levels and metastatic potential.

\section{Materials and methods}

Cell culture. MCF-7 and MDA-MB-231 cells were purchased from the cell bank of the Chinese Academy of Sciences (Shanghai, China). MDA-Kb2 cells were purchased from Shanghai Composite Biology Co., Ltd (http://www.xiangbio .com/; Shanghai, China). Normal human breast tissues were donated by the First Affiliated Hospital of Fujian Medical University (Fujian, China). Additional instruments and reagents used are in Table $\mathrm{I}$.

MDA-MB-231 and MDA-Kb2 cells were cultured in RPMI-1640 medium (Invitrogen; Thermo Fisher Scientific, Inc., Waltham, MA, USA) containing $10 \%$ fetal bovine serum (FBS; Thermo Fisher Scientific, Inc.) and MCF-7 cells were cultured in DMEM (Invitrogen; Thermo Fisher Scientific, Inc.) containing $0.01 \mathrm{mg} / \mathrm{ml}$ bovine insulin and $10 \% \mathrm{FBS}$. All cells were cultured in a humidified incubator with $5 \% \mathrm{CO}_{2}$ at $37^{\circ} \mathrm{C}$.

Immunofluorescence. Cells were fixed with $4 \%$ paraformaldehyde at room temperature for $30 \mathrm{~min}$, permeabilized with $0.05 \%$ Triton X-100 and blocked with $1 \%$ bovine serum albumin (Invitrogen; Thermo Fisher Scientific, Inc.) for $30 \mathrm{~min}$ at room temperature. The cells were subsequently washed using $1 \mathrm{X}$ Tween and PBS (PBST) solution (Beyotime Institute of Biotechnology, Haimen, China) and incubated with anti-HMGB1 (cat. no. ab18256; 1:50; Abcam, Cambridge, UK), anti-TLR4 (cat. no. ab22048; 1:50; Abcam) and anti-MyD88 (cat. no. 9284; 1:50; Cell Signaling Technology, Inc., Danvers, MA, USA) monoclonal antibodies for $1 \mathrm{~h}$ at room temperature and washed five times with $1 \mathrm{X}$ PBST, followed by incubation with Alexa Fluor 555 labeled donkey anti-mouse immunoglobulins (1:100; cat. no. A0460; Beyotime Institute of Biotechnology, Shanghai, China) for $1 \mathrm{~h}$ at room temperature. The cells were treated with DAPI (Sigma-Aldrich; Merck KGaA, Darmstadt, Germany) for $15 \mathrm{~min}$ at room temperature in dark to stain nuclei. Fluorescence images (x400) were captured using an Olympus confocal scanning microscope (Olympus Corporation, Tokyo, Japan). The relative fluorescence intensity was calculated with Image J densitometry software (version 1.6, National Institutes of Health, Bethesda, MD, USA).

Immunohistochemistry. A total of 100 tissue samples were collected from patients with breast cancer of different stages from the specimen repository of the Department of Breast and Thyroid Surgery, The First Affiliated Hospital of Fujian Medical University. These samples were initially obtained between January 2012 and December 2013, and were documented for age, family cancer history, tumor size, histological grade, tumor stage and axillary lymph node metastasis. A total of 20 cancer-adjacent tissues were used as a control.

All patients were women aged $35-70$ years, with a median age of 44 years, and all gave written informed consent. Ethical approval was obtained from the ethics committee of The First Affiliated Hospital of Fujian Medical University [(2014)106]. None of the patients received neoadjuvant chemotherapy or radiotherapy prior to biopsy, although each patient received individualized adjuvant chemotherapy. The tumors were staged per the American Joint Cancer Committee Guidelines (31) and included 21 cases of Stage I disease, 38 cases of Stage II disease and four cases of Stage III disease. Histological grading was referred to as the standard of diagnosis and treatment (19).

Cancer and control tissues were used for HMGB1, TLR4 and Myd88 protein detection. Antibodies used for immunohistochemical staining included anti-HMGB1 (cat. no. ab18256; 1:50; Abcam), anti-TLR4 (cat. no. ab22048; 1:50; Abcam) and anti-MyD88 (cat. no. 9284; 1:50; Cell Signaling Technology, Inc.). Briefly, tissue slides were deparaffinized in xylene and rehydrated with an ethanol gradient, consisting of 5-minute washes using absolute 95, 80 and 70\% ethanol. Then the sections were subjected to antigen retrieval by boiling in $0.01 \mathrm{~mol} / 1$ sodium citrate buffer ( $\mathrm{pH}$ 6.0) in a microwave oven for $10 \mathrm{~min}$. Following blocking of endogenous peroxidase activity with $0.3 \%$ hydrogen peroxide and blocking nonspecific protein binding with $1.5 \%$ normal goat serum (Thermo Fisher Scientific, Inc.) at room temperature for $1 \mathrm{~h}$ the sections were incubated overnight with primary antibodies at $4^{\circ} \mathrm{C}$ in a humidified chamber. The sections were subsequently incubated with biotinylated goat anti-mouse IgG (cat. no. ab64255; Abcam) for $30 \mathrm{~min}$ at $37^{\circ} \mathrm{C}$ and proteins were detected with 3,3'-diaminobenzidine. To evaluate the presence or absence of lung metastasis, the lung tissues were serially cut into 5- $\mu \mathrm{m}$ slices and every 10 th section was stained with hematoxylin for $10 \mathrm{~min}$, rinsed with running water, differentiated with hydrochloric acid and then stained with eosin for $5 \mathrm{~min}$ at room temperature The number of metastases in the lungs was calculated by two independent pathologists.

RNA isolation and reverse transcription-polymerase chain reaction $(R T-P C R)$. Total RNA was isolated from cells using TRIzol $^{\circledR}$ reagent (Invitrogen; Thermo Fisher Scientific, Inc.), according to the manufacturer's protocol. Total RNA (2 mg) was reverse transcribed to cDNA with PrimeScript ${ }^{\mathrm{TM}} 1$ st Strand 
cDNA Synthesis kit (Takara Biotechnology Co., Ltd., Dalian, China) and RT-PCR was carried out with TaKaRa Ex Taq (Takara Biotechnology Co., Ltd.). RT-PCR was performed according to the following conditions: Pre-denaturation at $95^{\circ} \mathrm{C}$ for $5 \mathrm{~min}$, followed by 35 cycles of $95^{\circ} \mathrm{C}$ for $1 \mathrm{~min}, 60^{\circ} \mathrm{C}$ for $45 \mathrm{sec}$ and $72^{\circ} \mathrm{C}$ for $45 \mathrm{sec}$, and a final extension at $72^{\circ} \mathrm{C}$ for $10 \mathrm{~min}$. The primers for RT-PCR were as follows: TLR4 forward (F), 5'-AATGGATCAAGGACCAGAGG-3' and reverse (R), 5'-CAGCCAGCAAGAAGCATCAG-3'; MYD88 F, 5'-CGC CGGATGGTGGTGGTTGT-3' and R, 5'-TGTAGTCGCAGA CAGTGATGAACC-3'; HMGB1 F, 5'-AATACGAAAAGG ATATTGCT-3' and R, 5'-GCGCTAGAACCAACTTAT-3'; and GAPDH F, 5'-ATCTGGCACCACACCTTCTACAATGAG CTGCG-3' and R, 5'-CGTCATCCCTGCTTGCTGATCCAC ATCTGC-3'. The relative mRNA expression of target genes was normalized to GAPDH with the method of $2^{-\Delta \Delta \mathrm{Cq}}(32)$.

Western blot analysis. Whole cell lysates were prepared using radioimmunoprecipitation assay buffer [1\% Triton X-100, $150 \mathrm{mmol} / 1 \mathrm{NaCl}, 1 \mathrm{mmol} / 1$ EGTA, $50 \mathrm{mmol} / 1$ Tris-HCl, $0.1 \%$ sodium dodecyl sulfate (SDS), $1 \%$ sodium deoxycholate and phenylmethylsuphonyl fluoride; Cell Signaling Technology, Inc.] and western blotting was performed as previously described (33). The concentration of protein was determined by bicinchoninic acid kit (Sigma-Aldrich, Merck $\mathrm{KGaA})$. The same amount of proteins $(30 \mu \mathrm{g})$ were separated by $12.5 \%$ SDS-PAGE and transferred onto polyvinylidene difluoride membranes. Following being blocked with 5\% non-fat milk at room temperature for $1 \mathrm{~h}$, the membranes were treated with anti-TLR4 (cat. no. ab22048; 1:100; Abcam) and anti-MyD88 (cat. no. 9284; 1:100; Cell Signaling Technology, Inc.). Antibodies at $4^{\circ} \mathrm{C}$ overnight. The next morning, the membranes were incubated with horseradish peroxidase (HRP)-conjugated goat anti-mouse secondary antibody (Santa Cruz Biotechnology, Inc.; cat. no. sc-2031; 1:10,000) for $2 \mathrm{~h}$ at room temperature. After being washed, bands were developed with enhanced chemiluminescence kit (Sigma-Aldrich, Merck $\mathrm{KGaA}$ ) and captured with Gel imaging system (Bio-Rad Laboratories, Inc., Hercules, CA, USA). The relative expression of target proteins was calculated with Quantity One Software V4.2 (Bio-Rad Laboratories, Inc.) normalized to GAPDH.

Statistical analysis. Data derived from at least three separate and independent experiments were expressed as the mean \pm standard deviation. Statistical differences of different groups were performed with SPSS version 19.0 (IBM Corp., Armonk, NY, USA). $\chi^{2}$ tests and the Fisher exact probability method were used to compare the differences between expression levels of HGBM1, TLR4 and MyD88 in each group, and the association between their expression levels and the clinicopathological features of breast cancer. A one-way analysis of variance with Dunnett's post-hoc analysis was used for comparisons between multiple groups. $\mathrm{P}<0.05$ was considered to indicate a statistically significant difference.

\section{Results}

Expression levels of TLR4, MYD88 and HMGB1 vary according to metastatic potential in breast cancer cells. It was identified that TLR4, MYD88 and HMGB1 were expressed 
to a marked extent in MDA-MB-231, MCF-7 and MDA-Kb2 cells. The respective expression levels of TLR4 and MYD88 in MDA-MB-231 cells were 10.43 and 2.09 times higher compared with those in $\mathrm{MCF}-7$ cells $(\mathrm{P}<0.05$; Fig. 1$)$. No significant differences were observed between the expression levels of either TLR4 or MYD88 in MCF-7 and MDA-Kb2 cells. TLR4 protein expression in MDA-MB-231 cells was 2.6 times higher compared with MCF-7 cells and 2.4 times higher compared with MDA-Kb2 cells. The protein expression levels of MYD88 in MDA-MB-231 cells were 1.6 times higher compared with MCF-7 cells and 1.8 times higher compared with MDA-Kb2 cells ( $\mathrm{P}<0.05$; Fig. 1). HMGB1 was also expressed in these three cell lines (Fig. 1).

The average fluorescence intensity of TLR4 protein expression in MDA-MB-231 cells was 0.14 and 0.08 in MCF-7 cells $(\mathrm{P}<0.01$; Fig. 2). There were no significant differences between the intensities in MCF-7 and MDA-Kb2 cells ( $\mathrm{P}>0.05)$. MyD88 protein was primarily expressed in the cytoplasm of all three cell types. The average fluorescence intensity of MyD88 in MDA-MB-231 and MCF-7 cells was 0.136 and 0.05 , respectively ( $\mathrm{P}<0.01$; Fig. 3), while there was no significant difference between the levels in MCF-7 and MDA-Kb2 cells ( $\mathrm{P}>0.05)$. These results suggested that an association may exist between TLR4 and MyD88 and the invasive potential of breast cancer cells.

Expression of TLR4 and MyD88 correlates with the metastatic potential of tumors from patients with breast cancer. To examine the association between TLR4/MyD88 signaling and breast cancer progression, breast tumors were collected from patients and used to detect the protein expression levels of TLR4 and MyD88. Randomly selected cancer-adjacent tissues were used as controls. It was identified that TLR4 was primarily localized to the plasma membrane as fine brown granules (Fig. 4). Expression of TLR4 was identified in $46 \%$ (46/100 cases) of breast tumors and $20 \%$ (4/20 cases) of cancer-adjacent tissues ( $<<0.001$; Table I). MyD88 was primarily localized to the cytoplasm as brownish yellow granules (Fig. 4). Expression of MyD88 was identified in 41\% (41/100 cases) of breast tumors and $25 \%$ (5/20 cases) of cancer-adjacent tissues $(\mathrm{P}<0.001$; Table I). These results were consistent with the expression patterns of TLR4 and MyD88 in vitro.

TLR4, MyD88 and HMGB1 protein expression is associated with poor prognosis in patients with breast cancer. To determine whether there was a correlation between the expression levels of TLR4, MyD88 and HMGB1 and breast cancer invasion, clinical and pathological data were collected from the 100 cases of breast cancer used in the expression analysis. It was identified that the expression rates of TLR4 in patients with or without axillary lymph node metastasis were 68.8 and $55 \%(\mathrm{P}<0.05)$, respectively. TLR4 expression rates for samples from patients with Stage I/II or Stage III disease were 33.8 and $71.9 \%(\mathrm{P}<0.001$; Table II), respectively. The expression rates of MyD88 in samples with or without axillary lymph nodes metastasis were 59 and 25.6\% $(\mathrm{P}<0.05)$, respectively. MyD88 expression rates in samples from patients with Stage I/II or Stage III disease were 44.1 and $65.6 \%$, respectively $(\mathrm{P}<0.05$; Table III). The expression rates of HMGB1 in samples with or without axillary lymph node metastasis were 63.9 and $41 \%(\mathrm{P}<0.05)$, respectively. For samples from patients with
Table II. TLR4 expression and the corresponding breast cancer clinical pathological features.

\begin{tabular}{lrrrrr}
\hline & \multicolumn{5}{c}{ TLR4 } \\
\cline { 3 - 4 } Characteristics & No. & - & + & $\chi^{2}$ & P-value \\
\hline Axillary lymph node & & & & & \\
metastasis & & & & & \\
$\quad$ Yes & 61 & 19 & 42 & 12.45 & 0.002 \\
$\quad$ No & 39 & 27 & 12 & & \\
Histological grade & & & & & \\
$\quad$ Yes & 68 & 45 & 23 & 8.155 & 0.006 \\
$\quad$ No & 32 & 9 & 23 & & \\
\hline
\end{tabular}

TLR4, Toll-like receptor 4.

Table III. MyD88 expression and the corresponding breast cancer clinical pathological features.

\begin{tabular}{llllll}
\hline & \multicolumn{5}{c}{ MyD88 } \\
\cline { 3 - 4 } Characteristics & No. & - & + & $\chi^{2}$ & P-value \\
\hline Axillary lymph & & & & & \\
node metastasis & & & & & \\
$\quad$ Yes & 61 & 25 & 36 & 6.094 & 0.015 \\
No & 39 & 29 & 10 & & \\
Histological grade & & & & & \\
$\quad$ Yes & 68 & 38 & 30 & 5.366 & 0.039 \\
No & 32 & 11 & 21 & & \\
\hline
\end{tabular}

MyD88, myeloid differentiation factor 88 .

Table IV. HMGB1 expression and the corresponding breast cancer clinical pathological features.

\section{HMGB 1}

$\begin{array}{lllll}\text { Characteristics } & \text { No. } \quad-\quad+ & \chi^{2} & \text { P-value }\end{array}$

\begin{tabular}{llllll}
\hline $\begin{array}{l}\text { Axillary lymph } \\
\text { node metastasis }\end{array}$ & & & & & \\
$\quad$ Yes & 61 & 22 & 39 & 6.994 & 0.017 \\
No & 39 & 23 & 16 & & \\
Histological & & & & & \\
grade & & & & & \\
Yes & 68 & 40 & 28 & 5.587 & 0.028 \\
No & 32 & 10 & 22 & & \\
\end{tabular}

HMGB1, high mobility group box 1 .

Stage I/II or Stage III disease, the rates were 41.2 and $68.7 \%$, respectively $(\mathrm{P}<0.05$; Table IV). 

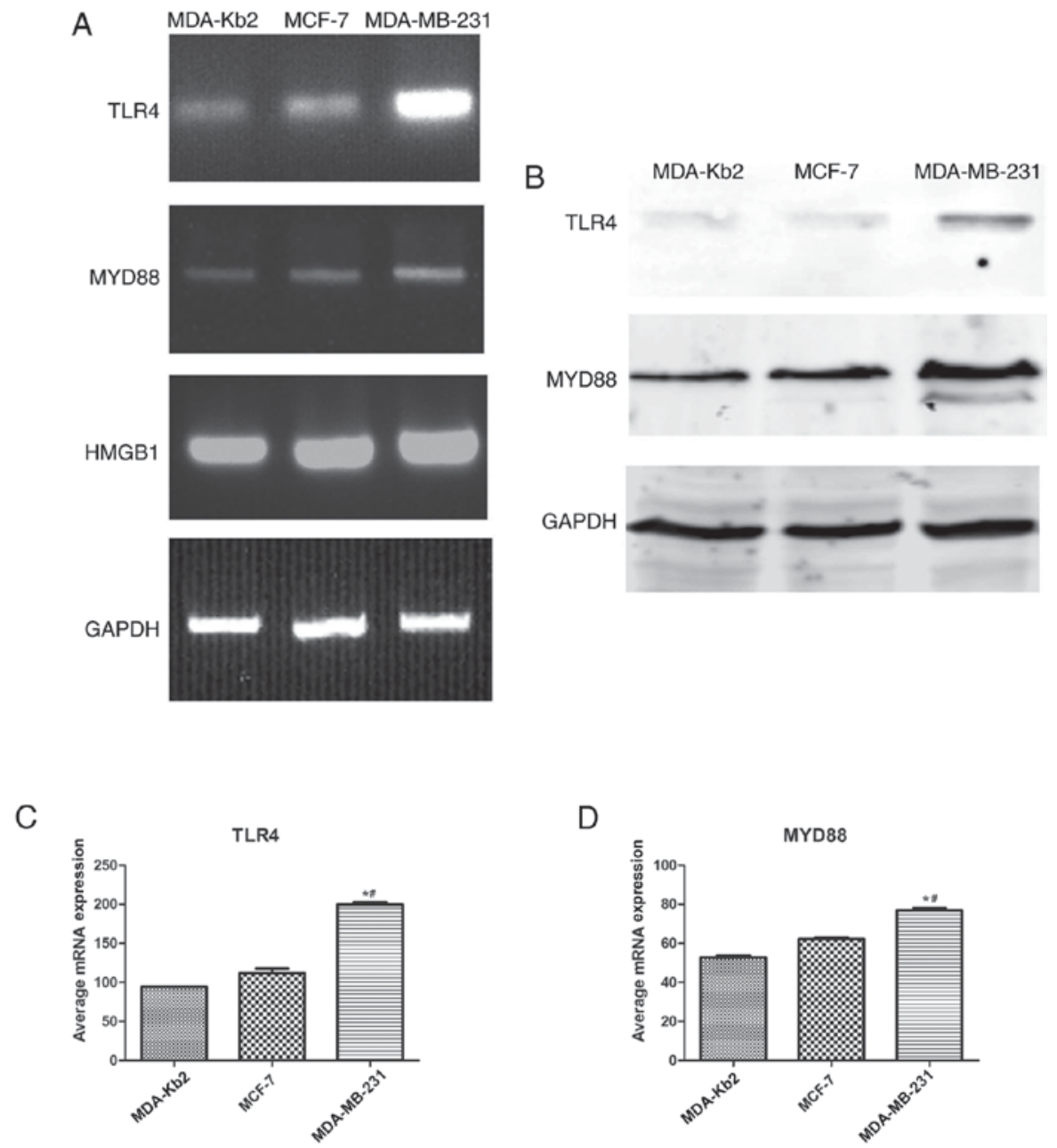

$\mathrm{D}$
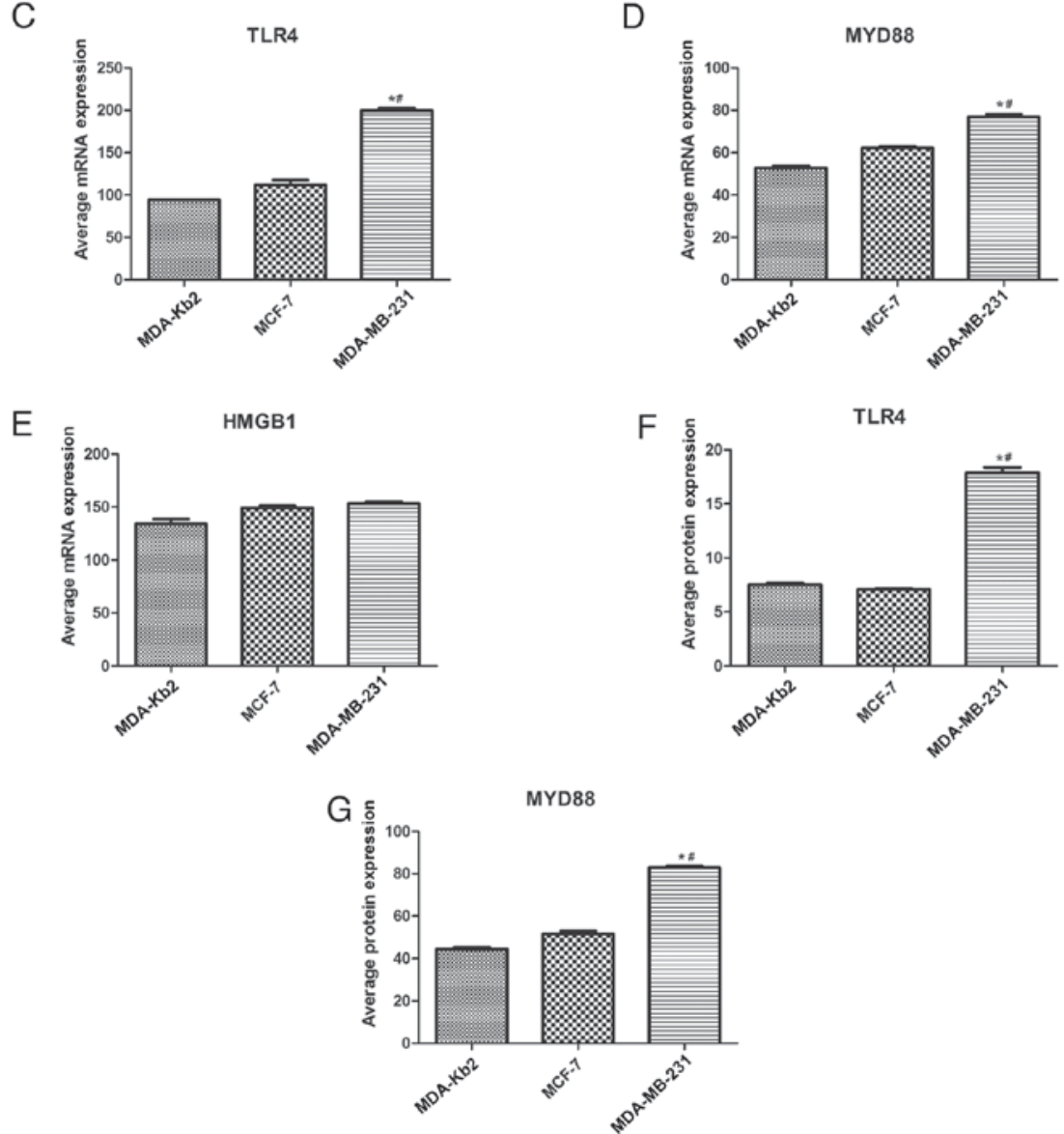

Figure 1. TLR4, MYD88 and HMGB1 mRNAs are expressed in MDA-MB-231, MCF-7 and MDA-Kb2 cells. (A) Total RNA was isolated from MDA-MB-231, MCF-7 and MDA-Kb2 cells for RT-PCR. (B) Proteins were extracted for western blot. The relative mRNA levels of TLR4 (C), MYD88 (D) and HMGB1 (E) were normalized to GAPDH. The relative protein levels of TLR4 (F) and MYD88 (G) were calculated by the normalization to GAPDH. "P<0.05. TLR4, Toll-like receptor 4; MyD88, myeloid differentiation factor 88; HMGB1, high mobility group box 1 .

\section{Discussion}

Breast cancer is one of the most common cancer types in women and is the second most common among all cancer types worldwide. Targeted medicine has become more widespread, as it improves the diagnosis, prognosis and treatment of patients with breast cancer (31). More effective molecular targets of carcinogenesis and metastasis in breast cancer are 

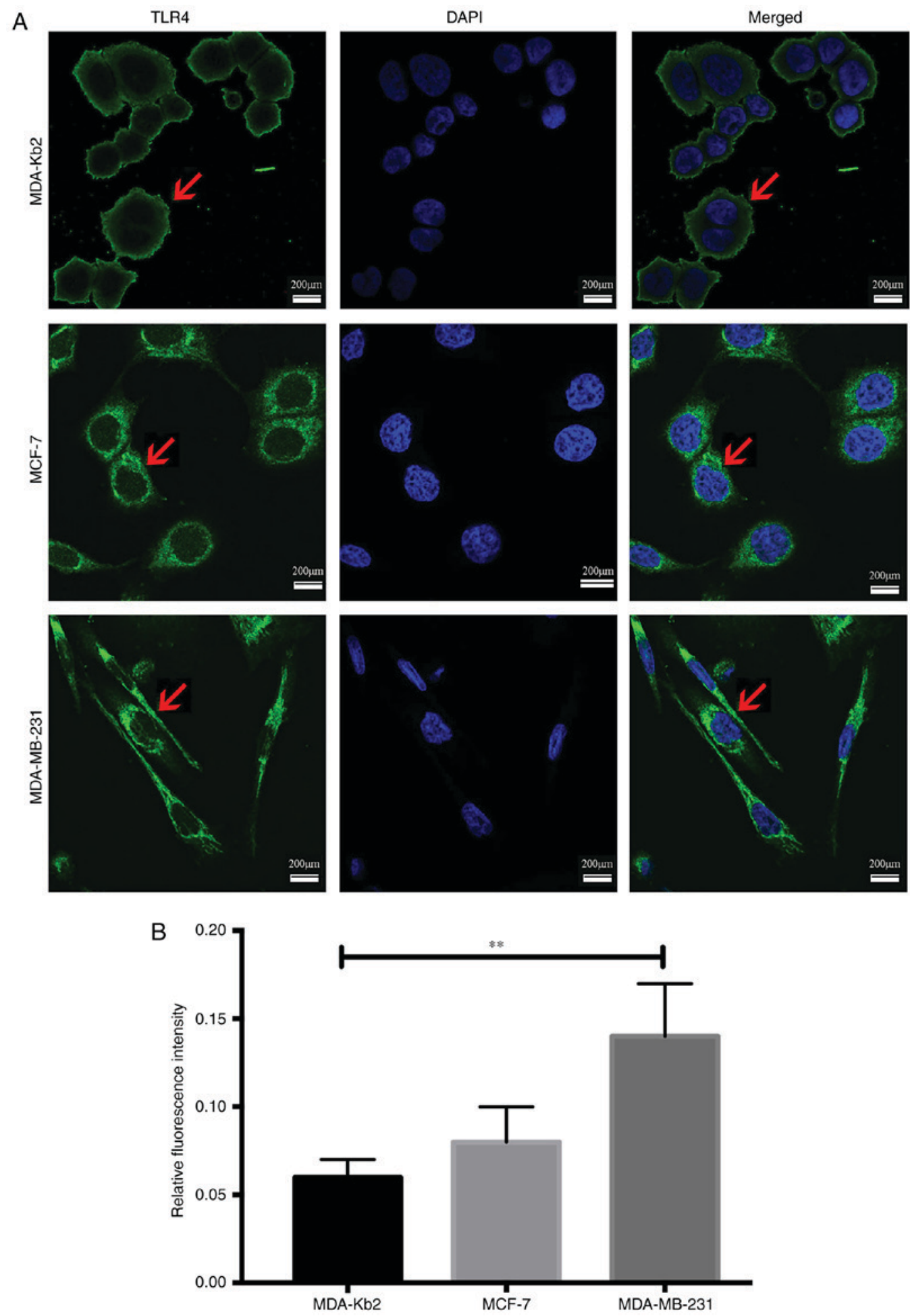

Figure 2. TLR4 protein expression in MDA-MB-231, MCF-7 and MDA-Kb2 cells. (A) The localization of TLR4 in MDA-MB-231, MCF-7 and MDA-Kb2 was determined by immunofluorescence. TLR4-positive cells were marked in green (indicated by the red arrow) and nuclear was stained with DAPI in blue. Scale bar=200 $\mu \mathrm{m}$. (B) The relative fluorescence intensity was calculated with ImageJ densitometry software. ${ }^{* *} \mathrm{P}<0.01$. TLR4, Toll-like receptor 4.

being identified every year, including erb-b2 receptor tyrosine kinase 2 (34), X-C motif chemokine receptor 1 (35) and the mTOR effectors ribosomal protein S6 kinases B1 and 2, and eukaryotic translation initiation factor $4 \mathrm{E}-$ binding protein 1 (36).

TLR4/MyD88 signaling occurs primarily during inflammation (37), and activation of the TLR4 complex may control the pathophysiology of a number of human diseases, including cardiovascular disorders, diabetes, metabolic syndrome, autoimmune disorders, neuroinflammatory disorders, autism and chronic fatigue syndrome (38). Repressing TLR4/MyD88 signaling decreases cell viability, activates apoptosis and increases the levels of inflammatory factors following Bacillus Calmette-Guerin infection in macrophages (39). Furthermore, ochratoxin DefiA induces immune-associated toxicity via reactive oxygen species-mediated TLR4/MyD88 signaling 
A
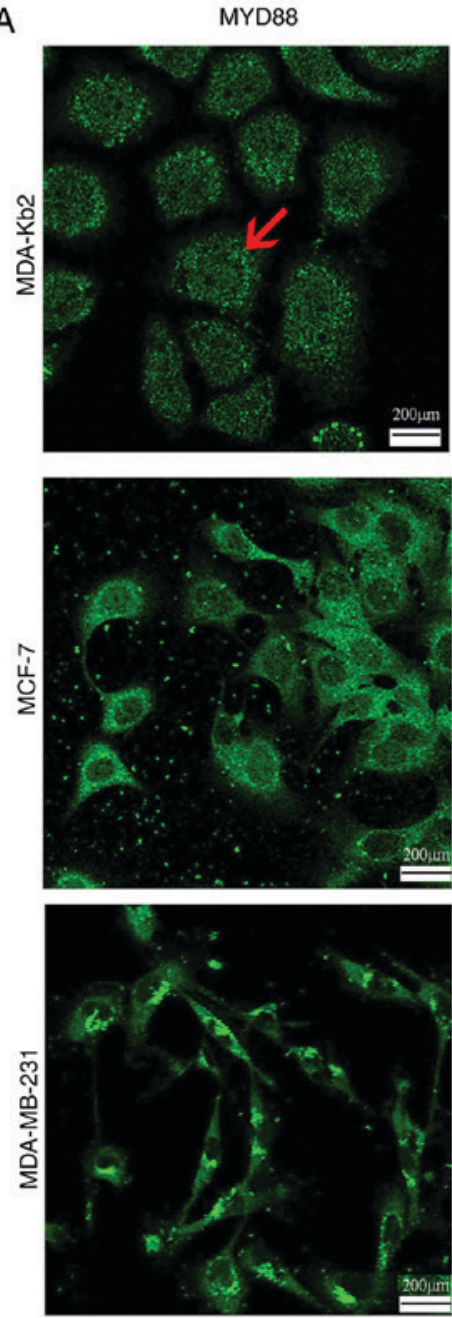

DAPI
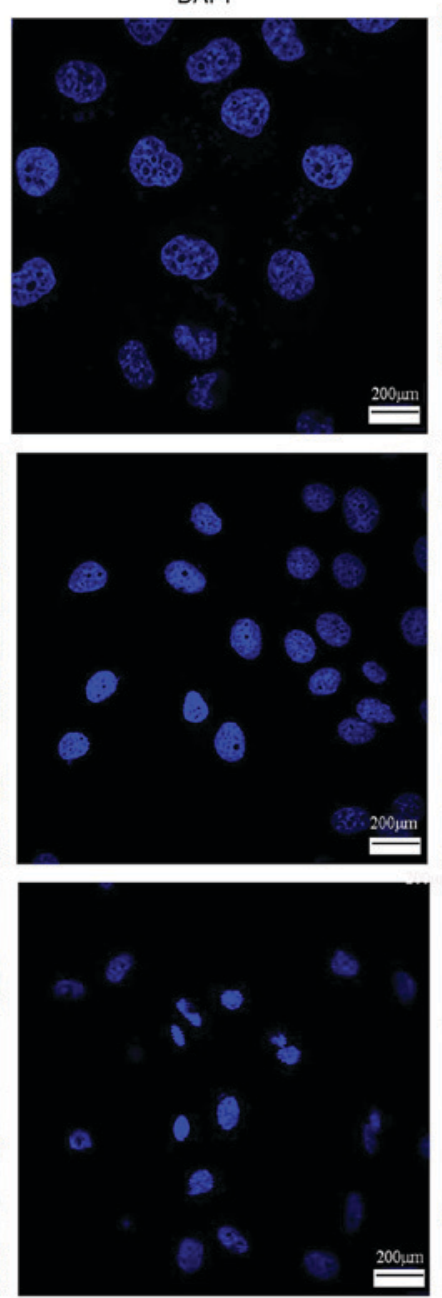

Merged
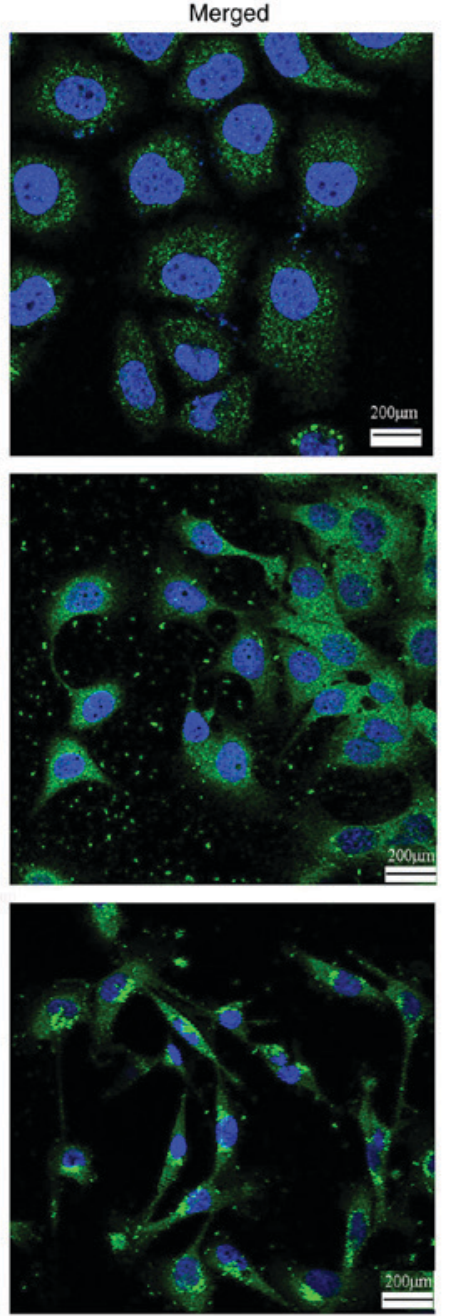

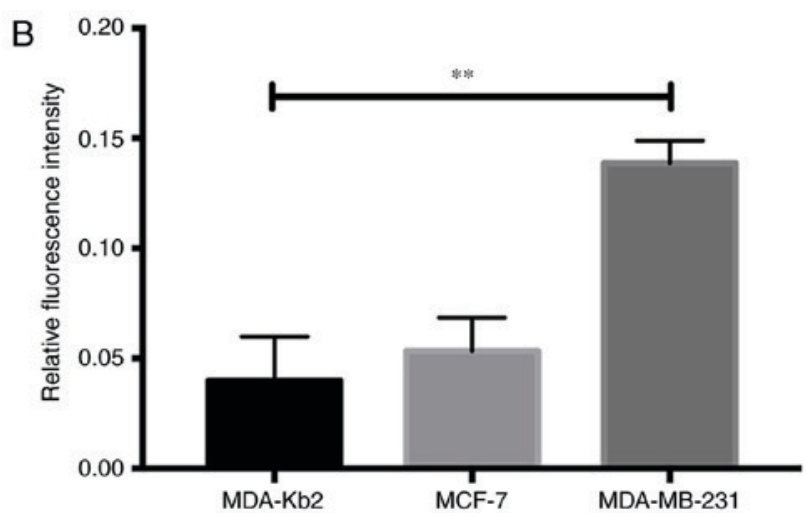

Figure 3. MyD88 protein expression in MDA-MB-231, MCF-7 and MDA-Kb2 cells. (A) The localization of MyD88 in MDA-MB-231, MCF-7 and MDA-Kb2 was determined by immunofluorescence. MYD88-positive cells were marked in green (indicated by red arrow) and nuclear was stained with DAPI in blue. Scale bar $=200 \mu \mathrm{m}$. (B) The relative fluorescence intensity was calculated with ImageJ densitometry software. ${ }^{* *} \mathrm{P}<0.01$. MyD88, myeloid differentiation factor 88 .

in porcine alveolar macrophages (40), and curcumin (41) and irisin (42) exert their biological functions by inhibiting TLR4/MyD88/NF- $\kappa$ B signaling. The tumor-associated inflammatory microenvironment may serve a pivotal role in the progression and prognosis of a number of cancer types, including ovarian, rectal and prostate cancer (43). Methicillin-resistant Staphylococcus aureus infection may enhance non-small cell lung cancer metastasis by upregulating
TLR4 signaling (44), and polysaccharopeptide exerts immunomodulatory effects through TLR4-TIRAP/MAL-MyD88 signaling in peripheral blood mononuclear cells from patients with breast cancer (45).

Cellular invasion is a common characteristic of malignant tumors. Tumor invasiveness is frequently accompanied by the overexpression and activation of oncogenes, or the loss of tumor suppressors. The estrogen receptor-positive 

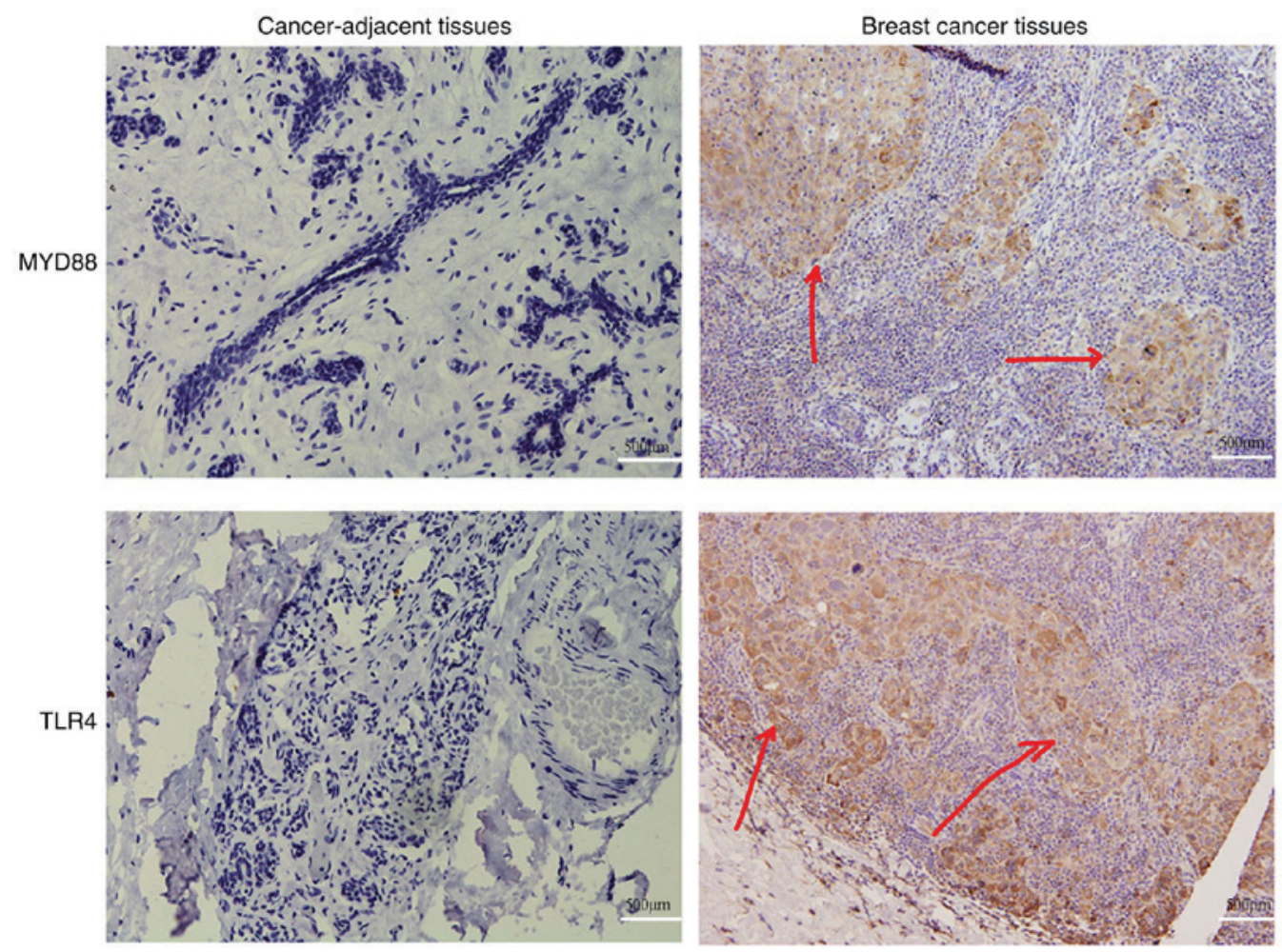

Figure 4. TLR4 and MyD88 protein expression levels in breast cancer and cancer-adjacent tissues. The expression of TLR4 (brown granules marked with red arrows) was primarily localized to the membrane. MyD88 expression (yellow granules marked with red arrows) was primarily localized to the cytoplasm. Scale bar $=500 \mu \mathrm{m}$. TLR4, Toll-like receptor 4; MyD88, myeloid differentiation factor 88.

human breast cancer cell line MCF-7, which has a low metastatic potential, is the most common cellular model of breast cancer. By contrast, MDA-MB-231, which is estrogen receptor-negative, has a high rate of invasion and spontaneous metastasis. The results of the present study demonstrated that HMGB1, TLR4 and MyD88 were expressed in MCF-7, MDA-MB-231 and MDA-Kb2 cells. It was identified that the protein and mRNA expression levels of TLR4 and MyD88 were significantly higher in MDA-MB-231 cells compared with MCF-7 cells. These results demonstrated a positive association between TLR4/MyD88 expression and invasive potential, which is consistent with the results of other studies performed on colorectal and ovarian cancer $(18,46)$. However, the levels of HMGB1, the ligand of TLR4, demonstrated no statistical differences between cell lines, suggesting that the expression of the TLR4 receptor, and not its ligand, is the key regulatory factor that determines invasiveness.

To further examine the association between the TLR4/MyD88 pathway and breast cancer progression, the expression levels of TLR4 and MyD88 in breast tumors from patients with various stages of disease were assessed. The results demonstrated that the expression levels of TLR4 and MyD88 were significantly increased in breast tumors compared with normal breast tissue. These levels were positively correlated with axillary lymph node metastasis and histological grade. This observation confirmed the association between the TLR4/MyD88 pathway and breast cancer and may provide a novel potential biomarker and therapeutic target to aid in the prognosis and treatment of patients with this type of cancer.
In conclusion, the expression levels of TLR4/MyD88 were positively correlated with the metastatic potential of breast cancer cells and tumors. The expression levels of TLR4/MyD88 may be used as a biomarker to evaluate the prognosis and guide the treatment of patients with breast cancer.

\section{Acknowledgements}

Not applicable.

\section{Funding}

The present study was sponsored by the Key Clinical Specialty Discipline Construction Program of Fujian, P.R.C. Professor Development Fund (grant no. JS14021) and the Natural Science Foundation of Fujian Province (grant no. 2015J01386).

\section{Availability of data and materials}

All data generated or analyzed during this study are included in this published article.

\section{Authors' contributions}

KLW managed the study and the materials, and constructed the target breast cancer cell lines; HHZ was responsible for the choice of experimental materials and performed the IHC experiments; YJF examined the mRNA and protein levels of TLR; YZZ determined the localization of TLR4 in cells; LJK detected the expression of HMGB1, TLR4 and MyD88; LC 
assisted cell culture and data sorting; FZ performed MCF-7 and MDA-MB-231 cell culture and data sorting; LFY constructed and identified TLR-siRNA plasmid and transfected cells; and XJC supervised the project and determined the breast cancer clinical pathological features.

\section{Ethics approval and consent to participate}

Ethical approval was obtained from the ethics committee of The First Affiliated Hospital of Fujian Medical University [Fujian, China; (2014)106]. All patients gave written informed consent

\section{Patient consent for publication}

All patients were given written informed consent. Ethical approval was obtained from the ethics committee of The First Affiliated Hospital of Fujian Medical University [(2014)106].

\section{Competing interests}

The authors declare that they have no competing interests.

\section{References}

1. Siegel R, Ma J, Zou Z and Jemal A: Cancer statistics, 2014. CA Cancer J Clin 64: 9-29, 2014

2. Dall GV and Britt KL: Estrogen effects on the mammary gland in early and late life and breast cancer risk. Front Oncol 7: 110, 2017.

3. Zhou PT, Li B, Ji J, Wang MM and Gao CF: A systematic review and meta-analysis of the association between OGG1 Ser326Cys polymorphism and cancers. Med Oncol 32: 472, 2015.

4. Liu YL, Zhang HM, Pan HM, Bao YH, Xue J, Wang TC, Dong XC, Li XL and Bao HG: The relationship between apolipoprotein $\mathrm{E}$ gene $\varepsilon 2 / \varepsilon 3 / \varepsilon 4$ polymorphism and breast cancer risk: A systematic review and meta-analysis. Onco Targets Ther 9: $1241-1249,2016$

5. Falzon C, Radel R, Cantor A and d'Arripe-Longueville F: Understanding narrative effects in physical activity promotion: The influence of breast cancer survivor testimony on exercise beliefs, self-efficacy, and intention in breast cancer patients. Support Care Cancer 23: 761-768, 2015.

6. Morrow RJ, Etemadi N, Yeo B and Ernst M: Challenging a misnomer? The role of inflammatory pathways in inflammatory breast cancer. Mediators Inflamm 2017: 4754827, 2017.

7. Jiang A, Zhao H, Cai J and Jiang WG: Possible effect of muscle-relaxant anaesthetics on invasion, adhesion and migration of breast cancer cells. Anticancer Res 36: 1259-1265, 2016.

8. Chen JC, Chang NW, Chung JG and Chen KC: Saikosaponin-A induces apoptotic mechanism in human breast MDA-MB-231 and MCF-7 cancer cells. Am J Chin Med 31: 363-377, 2003.

9. Yang S, Zhou Q and Yang X: Caspase-3 status is a determinant of the differential responses to genistein between MDA-MB-231 and MCF-7 breast cancer cells. Biochim Biophys Acta 1773: 903-911, 2007.

10. El Guerrab A, Cayre A, Kwiatkowski F, Privat M, Rossignol JM, Rossignol F, Penault-Llorca F and Bignon YJ: Quantification of hypoxia-related gene expression as a potential approach for clinical outcome prediction in breast cancer. PLoS One 12: e0175960, 2017.

11. Noh WC, Kim YH, Kim MS, Koh JS, Kim HA, Moon NM and Paik NS: Activation of the mTOR signaling pathway in breast cancer and its correlation with the clinicopathologic variables. Breast Cancer Res Treat 110: 477-483, 2008.

12. Zhou Y, Kipps TJ and Zhang S: Wnt5a signaling in normal and cancer stem cells. Stem Cells Int 2017: 5295286, 2017.

13. Boyault S, Drouet Y, Navarro C, Bachelot T, Lasset C, Treilleux I, Tabone E, Puisieux A and Wang Q: Mutational characterization of individual breast tumors: TP53 and PI3K pathway genes are frequently and distinctively mutated in different subtypes. Breast Cancer Res Treat 132: 29-39, 2012.
14. Mandal A, Bhatia D and Bishayee A: Anti-inflammatory mechanism involved in pomegranate-mediated prevention of breast cancer: The role of NF- $\mathrm{KB}$ and Nrf2 signaling pathways. Nutrients 9: E436, 2017.

15. Orlowski RZ and Dees EC: The role of the ubiquitination-proteasome pathway in breast cancer: Applying drugs that affect the ubiquitin-proteasome pathway to the therapy of breast cancer. Breast Cancer Res 5: 1-7, 2003.

16. Castrellon AB: Novel strategies to improve the endocrine therapy of breast cancer. Oncol Rev 11: 323, 2017.

17. Kadowaki N, Ho S, Antonenko S, Malefyt RW, Kastelein RA, Bazan F and Liu YJ: Subsets of human dendritic cell precursors express different toll-like receptors and respond to different microbial antigens. J Exp Med 194: 863-869, 2001.

18. Li Z, Block MS, Vierkant RA, Fogarty ZC, Winham SJ, Visscher DW, Kalli KR, Wang C and Goode EL: The inflammatory microenvironment in epithelial ovarian cancer: A role for TLR4 and MyD88 and related proteins. Tumour Biol 37: 13279-13286, 2016.

19. Harberts E, Zhou H, Fishelevich R, Liu J and Gaspari AA: Ultraviolet radiation signaling through TLR4/MyD88 constrains DNA repair and plays a role in cutaneous immunosuppression. J Immunol 194: 3127-3135, 2015.

20. d'Adhemar CJ, Spillane CD, Gallagher MF, Bates M, Costello KM, Barry-O'Crowley J, Haley K, Kernan N, Murphy C, Smyth PC, et al: The MyD88+ phenotype is an adverse prognostic factor in epithelial ovarian cancer. PLoS One 9: e100816, 2014.

21. Huang JM, Zhang GN, Shi Y, Zha X, Zhu Y, Wang MM, Lin Q, Wang W, Lu HY, Ma SQ, et al: Atractylenolide-I sensitizes human ovarian cancer cells to paclitaxel by blocking activation of TLR4/MyD88-dependent pathway. Sci Rep 4: 3840, 2014.

22. Echizen K, Hirose O, Maeda Y and Oshima M: Inflammation in gastric cancer: Interplay of the COX-2/prostaglandin E2 and Toll-like receptor/MyD88 pathways. Cancer Sci 107: 391-397, 2016.

23. Gnanasekar M, Kalyanasundaram R, Zheng G, Chen A, Bosland MC and Kajdacsy-Balla A: HMGB1: A promising therapeutic target for prostate cancer. Prostate Cancer 2013: 157103, 2013.

24. Liu W, Zhang Z, Zhang Y, Chen X, Guo S, Lei Y, Xu Y, Ji C, Bi Z and Wang K: HMGB1-mediated autophagy modulates sensitivity of colorectal cancer cells to oxaliplatin via MEK/ERK signaling pathway. Cancer Biol Ther 16: 511-517, 2015.

25. Zhang QY, Wu LQ, Zhang $T$, Han $Y F$ and Lin $X$ : Autophagy-mediated HMGB1 release promotes gastric cancer cell survival via RAGE activation of extracellular signal-regulated kinases 1/2. Oncol Rep 33: 1630-1638, 2015.

26. Dong YD, Cui L, Peng CH, Cheng DF, Han BS and Huang F. Expression and clinical significance of HMGB1 in human liver cancer: Knockdown inhibits tumor growth and metastasis in vitro and in vivo. Oncol Rep 29: 87-94, 2013.

27. Feng $\mathrm{A}, \mathrm{Tu} \mathrm{Z}$ and Yin B: The effect of HMGB1 on the clinicopathological and prognostic features of non-small cell lung cancer. Oncotarget 7: 20507-20519, 2016.

28. Chen X, Zhao F, Zhang H, Zhu Y, Wu K and Tan G: Significance of TLR4/MyD88 expression in breast cancer. Int J Clin Exp Pathol 8: 7034-7039, 2015.

29. Gkretsi V, Stylianou A, Louca M and Stylianopoulos T: Identification of Ras suppressor-1 (RSU-1) as a potential breast cancer metastasis biomarker using a three-dimensional in vitro approach. Oncotarget 8: 27364-27379, 2017.

30. Sun N, Xu HN, Luo Q and Li LZ: Potential indexing of the invasiveness of breast cancer cells by mitochondrial redox ratios. Adv Exp Med Biol 923: 121-127, 2016.

31. Denkert C, Liedtke C, Tutt A and von Minckwitz G: Molecular alterations in triple-negative breast cancer-the road to new treatment strategies. Lancet 389: 2430-2442, 2017.

32. Livak KJ and Schmittgen TD: Analysis of relative gene expression data using real-time quantitative PCR and the 2(-Delta Delta C(T)) method. Methods 25: 402-408, 2001.

33. Kurien BT and Scofield RH: Western blotting: An introduction. Methods Mol Biol 1312: 17-30, 2015.

34. Milanezi F, Leitão D, Ricardo S, Augusto I and Schmitt F: Evaluation of HER2 in breast cancer: Reality and expectations. Expert Opin Med Diagn 3: 607-620, 2009.

35. Yang XL, Qi LG, Lin FJ and Ou ZL: The role of the chemokine receptor XCR1 in breast cancer cells. Breast Cancer (Dove Med Press) 9: 227-236, 2017. 
36. Karlsson E, Pérez-Tenorio G, Amin R, Bostner J, Skoog L, Fornander T, Sgroi DC, Nordenskjöld B, Hallbeck AL and Stål O: The mTOR effectors 4EBP1 and S6K2 are frequently coexpressed, and associated with a poor prognosis and endocrine resistance in breast cancer: A retrospective study including patients from the randomised Stockholm tamoxifen trials. Breast Cancer Res 15: R96, 2013.

37. TotsukaN,Kim YG,KanemaruK,NiizumaK,UmemotoE,Nagai K, Tahara-Hanaoka S, Nakahasi-Oda C, Honda S, Miyasaka M, et al: Toll-like receptor 4 and MAIR-II/CLM-4/LMIR2 immunoreceptor regulate VLA-4-mediated inflammatory monocyte migration. Nat Commun 5: 4710, 2014.

38. Lucas K and Maes M: Role of the Toll Like receptor (TLR) radical cycle in chronic inflammation: Possible treatments targeting the TLR4 pathway. Mol Neurobiol 48: 190-204, 2013.

39. Xue X, Qiu Y and Yang HL: Immunoregulatory role of MicroRNA-21 in macrophages in response to bacillus calmette-guerin infection involves modulation of the TLR4/MyD88 signaling pathway. Cell Physiol Biochem 42: 91-102, 2017.

40. Xu H, Hao S, Gan F, Wang H, Xu J, Liu D and Huang K: In vitro immune toxicity of ochratoxin A in porcine alveolar macrophages: A role for the ROS-relative TLR4/MyD88 signaling pathway. Chem Biol Interact 272: 107-116, 2017.

41. Rahimifard M, Maqbool F, Moeini-Nodeh S, Niaz K, Abdollahi M, Braidy N, Nabavi SM and Nabavi SF: Targeting the TLR4 signaling pathway by polyphenols: A novel therapeutic strategy for neuroinflammation. Ageing Res Rev 36: 11-19, 2017.
42. Mazur-Bialy AI, Pocheć E and Zarawski M: Anti-inflammatory properties of irisin, mediator of physical activity, are connected with TLR4/MyD88 signaling pathway activation. Int J Mol Sci 18: E701, 2017.

43. Apetoh L, Ghiringhelli F, Tesniere A, Obeid M, Ortiz C, Criollo A, Mignot G, Maiuri MC, Ullrich E, Saulnier P, et al: Toll-like receptor 4-dependent contribution of the immune system to anticancer chemotherapy and radiotherapy. Nat Med 13: 1050-1059, 2007.

44. An J, Li Z, Dong Y, Ren J and Guo K: Methicillin-resistant staphylococcus aureus infection exacerbates NSCLC cell metastasis by up-regulating TLR4/MyD88 pathway. Cell Mol Biol (Noisy-le-grand) 62: 1-7, 2016.

45. Wang J, Dong B, Tan Y, Yu S and Bao YX: A study on the immunomodulation of polysaccharopeptide through the TLR4-TIRAP/MAL-MyD88 signaling pathway in PBMCs from breast cancer patients. Immunopharmacol Immunotoxicol 35: 497-504, 2013.

46. Aviello G, Corr SC, Johnston DG, O'Neill LA and Fallon PG: MyD88 adaptor-like (Mal) regulates intestinal homeostasis and colitis-associated colorectal cancer in mice. Am J Physiol Gastrointest Liver Physiol 306: G769-G778, 2014. International (CC BY-NC-ND 4.0) License. 